\title{
Visual Search Strategies of Tag Clouds - Results from an Eyetracking Study
}

\author{
Johann Schrammel ${ }^{1}$, Stephanie Deutsch ${ }^{1}$, and Manfred Tscheligi1,2 \\ ${ }^{1}$ CURE - Center for Usability Research and Engineering \\ ${ }^{2}$ University of Salzburg, ICT\&S Center \\ \{schrammel, deutsch, tscheligi\}@cure.at
}

\begin{abstract}
Tag clouds have become a frequently used interaction technique in the web in the past couple of years. Research has shown the influence of variables such as tag size and location on the perception of tag clouds. However, several questions remain unclear. First, little is know on how tag clouds are perceived visually and which search strategies users apply when looking for tags in a tag cloud. Second, there are variables, especially tag location, were prior work comes to conflicting results. Third, several approaches to present tag clouds with the tags semantically clustered have been proposed recently. However, it remains unclear which effects these new approaches have on the perception of tag clouds. In this paper we report the results of an extensive study on the perception of tag clouds using eye tracking technology that allows answering these questions.
\end{abstract}

Keywords: Tag clouds, eye tracking, folksonomy, clustering, semantic grouping, visualization.

\section{Introduction}

A tag cloud is a visual depiction of words, called tags, which is typically used to describe the contents of a web service. It consists of a visually weighted list of tags arranged in a certain layout. Tags are usually hyperlinks in form of single words that lead to a collection of more elaborated issues. The weight corresponding to their relevance or popularity within a context is characterized by one or more visual features for each word, such as font size, color, intensity, position or a combination of such features. The most used layout strategy is alphabetical sorting of tags, but also other principles such as importance-based or spatially optimized have been proposed and used. Recently semantic layouts, i.e. the clustering and display of tags according to their semantic relatedness, have attracted a lot of attention. Tag clouds have been used for various tasks such as searching, browsing, recognizing and impression formation about a subject [1].

Besides the evaluation of the importance of different visual features only little empirical evidence is available on the effectiveness of tag clouds. Several user-based studies on different aspects of tag clouds such as the interaction between different properties in tag cloud design are available that use traditional measurement methods 
of human performance (e.g. reaction time, accuracy). However, we are not aware of any investigations focusing on the visual perception of tag clouds that involved measurements with physiological methods, such as the recording of eye movements during stimulus presentation. We think eye tracking applications are particularly interesting as they allow interdisciplinary insights into the visual, cognitive, and attentional aspects of human information processing, for an example of use see [2]. Within HCI and Usability research eye movement recordings can provide an objective source of interface evaluation data.

\section{Related Work}

\subsection{Tag Clouds}

The influence and importance of visual features of tags in tag clouds has been the subject of several studies $[1,3,4,5]$. These studies provide concise evidence that the visual features font size, font weight and intensity seem to be the most important variables in affecting the perception of tag clouds. However, the exact role of tag position is not clear yet. Bateman et al. [4] reported no influence of the tag position on performance whereas Rivadeneira et al. [1] report that tags in the upper left quadrant of the tag cloud were recalled more frequently by their participants than tags placed in the lower-right quadrant. A similar focus on the upper-left quadrant was found by Halvey and Keane [3] for specific search tasks.

Words in tag clouds are typically listed in alphabetical order from upper-left to lower-right. However, recently several proposals for semantic arrangment of tags in a cloud were made. A layout algorithm based on tag similarity was proposed by Hassan-Montero and Herrero-Solana [6]. Fujimura et al. [7] use the cosine similarity of tag feature vectors (terms and their weight generated from a set of tagged documents) to measure tag similarity and then calculate a tag layout where the semantic relatedness is represented as the distance between tags. Berlocher et al. [8] propose a very similar approach.

Whereas the creators of these proposals clearly expect advantages for the interaction it remains questionable whether such improvements actually can be achieved. Schrammel et al. [5] could not find any substantial gains in performance for search tasks as well as for tag rememberability when they compared semantic layouts to tag clouds ordered alphabetically or randomly. Only for specific searches semantic layouts could provide an improvement over random organization, but as alphabetical layouts are still better than semantics for this type of task the actual usefulness of this approach remains questionable.

The usage of tag clouds have to consider different tasks and task contexts. For the application of tag clouds in search contexts Sinclair and Cardew-Hall [9] showed that people prefer traditional search interfaces over tag clouds to look for specific information. Tag cloud interfaces were preferred for the retrieving of more general information contents, so after all they remain to serve users as supplementary tools in search tasks, not as sole alternative to traditional search interfaces. Similarly the participants in the study of Kuo et al. [10] were able to resolve descriptive retrieval tasks better and faster from tag cloud interfaces than from queries of hierarchical ranked data, in 
this regard they rated tag clouds as more helpful. Concerning the identification of relationships between multiple concepts users gave better answers when using a search engine than with tag clouds. Overall users spent more time and ranked tag cloud interfaces less helpful as they did for a traditional search engine.

These results point out certain inappropriateness of tags clouds for use in specific search tasks which for our experiment led us to include two different types of tasks: specific search, i.e. finding a given tag in a tag cloud, and general search, i.e. finding a tag within a tag cloud that belongs to a specific topic.

\subsection{Basic Characteristics of Gaze Behavior}

Within eye tracking research, variables that appear to be valid indicators of visual perceptive processes are fixations, saccades, and scanpaths [11]. Eye fixation is defined as the spatially stable gaze on a specific area of the visual field for approx. 200-300 milliseconds. Although their interpretation depends on the context, fixation represents the critical moment between states of attention and higher cognitive processes e.g. encoding information. Relevant for analysis should be the number of fixation overall, target fixation, fixation duration as indicators of complexity of the visual task, and time interval from stimulus onset to first fixation on the display [12]. Between two fixations a saccade represents the eye movement to the next viewing position and typically lasts for 20-35 milliseconds. During saccades there is no encoding activity, so no indication about visual processing can be earned, only number of saccades and regressive saccades (backtracking eye-movements) can indicate difficulties during encoding processing.

We need to make saccades because of the limitation of the eyes acuity. The visual field is typically divided into three regions: foveal, parafoveal, and peripheral. The acuity is very good in the fovea (the central $2^{\circ}$ of vision), is not nearly so good in the parafovea (out to $5^{\circ}$ on either side of fixation) and is even poorer in the periphery (the region beyond the parafovea). In our experimental setup (see section 'Apparatus' below for details) $2^{\circ}$ is equivalent to about 35 pixel on the screen or 4 letters of a tag in the smallest font size.

Although humans can easily decouple the locus of attention and eye gaze location in simple discrimination tasks [13], in complex information processing tasks such as reading, the link between the two is probably quite tight. For analyzing the perception of tag clouds we therefore can securely work with the hypothesis that the spot of fixation is identical with the spot of attention.

\subsection{Gaze Behavior in Related Tasks}

\section{Basic Visual Search}

Eye movements are influenced by textual and typographical variables e.g. the manipulation of visual features. According to Duchowski [14] the classical search theory of Treisman and Gelade from 1980 contains a simple model of the visual system with practical relevance for the examination of eye movements. In this model a pre-attentive subsystem analyses simple visual features over the entire visual field in parallel. Classical simple visual features are brightness, orientation, size (or spatial frequency), color, movement, probably curvature, and maybe more. 


\section{Reading}

According to Keith Rayner [11] when reading English eye fixations last about 200$250 \mathrm{~ms}$ and the mean saccade size corresponds to 7-9 letter spaces. Besides there is evidence that eye movements are influenced by textual and typographical variables, as important to note, fixation duration increases and saccade length decreases when a text becomes more difficult. Also factors such as the quality of print, line length, and letter spacing influence patterns of eye movements, perception respectively. Perceptual span in reading: for readers of alphabetical orthographies (e.g., English, French, and Dutch) the span extends from the beginning of the currently fixated word but no more than 3-4 letters to the left of fixation to about 14-15 letter spaces to the right of fixation.

\section{Looking at graphs}

Other studies focus on eye movement analysis when people view statistical graphs, maps, and images [11]. In order to detect the underlying cognitive processes of graph comprehension Carpenter \& Shah [15] recorded people's eye movements while they examined graphs showing complex interactions and claim for the occurrence of 'chunking' processes during encoding of graphical information.

\section{In the web}

Particularly interesting findings offers the usage of eye tracking methodologies within Web usability studies. Klöckner et al. [16] observed most of their participants performing a linear strategy while browsing search results in order to evaluate each result one by one before selecting a document to open. Further work on eye movement recordings and Web search is presented by Cutrell \& Guan [17] and Pan et al. [18]. Lastly there should be noted the relevance of using eye tracking methodologies when investigating the impact of advertisements on people in consumer research [19]. Facing the increase of online information platforms and online newspapers new paradigms have been generated where people deal with more complex scenes, e.g. when a web page contains textual and graphical content. For the analysis of eye movements from Web viewers that did not alternate between the text and picture part of an advertisement see Rayner et al. [20].

\section{Research Questions}

The main objective of the current study is to better understand the visual search behavior of users when interacting with tag clouds. We want to find out which search patterns users apply, if and when they change their search strategies and whether they adapt or change these strategies in the different layout conditions. We also want to see whether findings from previous tag cloud studies based on measures of task completion times or item rememberability can be reproduced and confirmed using eye tracking techniques or if new interpretations are needed. We especially want to contribute to clarify the up till now controversial aspect of tag position. The analysis of eye movement metrics could clarify if and when people become aware of how tags are organized in the visualization, concretely if and when people recognize a specific order in a given tag arrangement during a search task, and whether or not they adopt the their search strategies. 


\section{Method}

\section{Apparatus}

Eye movements of all participants were recorded with Tobii x-50 Eye Tracker (Tobii Technology AB, Danderyd, Sweden), a standalone eye tracking unit with stimulus setup on a display in front of the subject. The Tobii Eye Tracker uses near infrared diodes to generate reflection patterns on the corneas of the eye of the user. These reflection patterns are collected by a high-resolution camera with a large fieldof- view to finally calculate the gaze point on the screen. Recordings of time stamps and gaze positions relative to stimuli for each eye were collected with ClearView 2.7.1 analysis software. The fixation filter was set to a fixation radius of 30 pixels and minimum fixation duration of 100 milliseconds.

Subjects sat in front of the stimulus monitor at a distance of approximately $60 \mathrm{~cm}$ from the eye tracker. Stimuli were displayed on a 17 inch LCD screen with a resolution of 1024 by 768 pixels. Before task initiation eye movements of each subject were calibrated appropriately, respecting calibration settings of 5 points at normal type, medium speed, and medium dot size.

\section{Data manipulation and quality assurance}

Eye tracking data are prone to systematic errors, especially for vertical position as small changes in the distance of the participant to the stimulus can have noticeable effects. Therefore we applied a manual control and correction of data. After data acquisition fixation filtered data were exported from ClearView to the statistical software (SPSS v.16.0) in form of a sequential list of fixations and eye movement recordings. Using a gaze pattern tool of ClearView 2.7.1 recordings of each participant were explicitly examined regarding systematic deviations and completeness of tracked data. The task screens were test participants had to click on a 'Next'-button served as reverence points to estimate the quality of the data. In case of systematic deviations of the gaze points were corrected by adjusting the y-coordinates in SPSS.

The exact position on the screen in pixels of all words in the tag clouds within all conditions was measured and these coordinates ( $\mathrm{X}$ and $\mathrm{Y}$ ) where used to link the fixation data to an actual word (and its size).

We are very confident that our data is very accurate. A good indicator for this accuracy is that in most cases the target word is listed in as one of the last words fixated before the task was finished.

\section{Sample}

15 subjects ( 7 male, 8 female) were recruited from our internal participant database according to the following criteria: all participants used the internet frequently and had good English skills. Their sights were normal or corrected to normal.

\section{Stimuli}

The basic test content for the experiment, adopted from previous work [5], was formed by 304 popular tags taken from different thematic clusters of flickr. Tags were randomly assigned to content groups which formed the basic elements for the tag 
clouds. Each tag cloud consisted of 76 items arranged in 7 lines with 11 respectively 10 tags per line. Every tag cloud included 6 very big, 11 big, 22 small, and 37 very small tags. Tags were randomly assigned to one of these font size groups.

Three different layouts were used in the experiment:

- Alphabetic: Here tags were placed alphabetically starting at the top-left similar to reading text.

- Random: Tags were placed randomly on the tag by use of a random number generator.

- Semantic: We used the getrelated-function of flickrs API [21] to retrieve a list of the tags most related to each word within the tag cloud. Then based on the number of co-occurring related tags a measure for the relatedness of two tags was calculated. An alternating least-squares algorithm to perform multidimensional scaling (ALSCAL) [22] was used to compute a two dimensional arrangement of the tags. In the third step we used the value on the y-axis to form 7 groups of 11 resp. 10 tags each. Next tags within each group were sorted according to their value on the x-axis. The result provided an 11 times 7 arrangement that was used to generate the tag cloud.

Figure 1 on the next page shows three example tag clouds derived from the same content group i.e. they consist of exactly the same tags with the same size, only the ordering is different.

\section{Procedure}

After being instructed adequately, subjects started with the tests. A browser window opened where the participants had to read a short instruction indicating the nature of the task on the upcoming screen. Readiness for stimulus onset was confirmed via mouse click on a 'Next'-button. Once the target tag was found, participants had to click on it to proceed to the next item.

For each of the three layouts 12 search tasks with different targets within the same tag cloud were shown. Target tags were evenly distributed across font sizes and location (quadrants). Subjects had to solve two different types of tasks. In the first task condition participants were demanded to find a specific tag within a tag cloud as fast and accurately as possible. The second task was to find a tag that belongs to a specific topic. In all experiments test conditions where presented in different order using content sets and tag cloud layouts that counterbalanced position and learning effects.

\section{Scanning detection}

In order to extract possible scanning patterns from the recorded eye movements of the participants in our experiment an algorithm was implemented that differentiates between a person's serial scanning behavior and chaotic or random search patterns. Our algorithm is based on the reading detecting system of Campbell and Maglio [23]. The underlying algorithm is known as pooled evidence and allows reliable and accurate recognition of reading performance. As serial scanning - contrary to reading - also can occur from right to left the algorithm had to be adapted. 
animal auto beach black blue boat bouquet bride cake california cat classic cloud clouds coast couple cute dance dancing dress eyes family feet flowers food friends girl groom happy holiday labrador landscape letter

light love mailboX marriage nature nose ocean old pacific parcel party people pet pink portrait postcard postoffice puppies reception red reflection retriever rocks roses sand sea seaside shadow shore silhouette sky snow stamp summer sun sunset surf vacation water wave wedding white woman

a) Alphabetic

blue labrador black cloud food reflection shadow family sand nose girl letter marriage clouds california cake people pet animal roses silhouette parcel cat shore postcard wedding red couple wave cute retriever groom dress landscape pink water friends light auto sky portrait sun holiday happy white bride old pacific party snow rocks sunset beach seaside dancing dance coast ocean stamp sea surf feet reception eyes love woman bouquet flowers nature puppies $\mathrm{MailboX}$ vacation postoffice summer boat classic b) Random

bouquet silhouette landscape sun couple love cat roses pink classic retriever cake food clouds nose feet dancing family eyes blue dance marriage cute groom Cloud nature dress old light parcel white flowers black reception reflection pet happy stamp woman postoffice summer california coast bride

girl shadow people wave water red sea pacific shore ocean labrador postcard snow vacation wedding friends rocks party letter surf beach seaside mailbox portrait puppies sky auto holiday sunset animal sand boat

\section{c) Semantic}

Fig. 1. Example content displayed in the three different layouts

Initially the system is in 'chaotic-search' mode until an event assigned to a specific eye movement occurs. Via incrementing or de-incrementing a 'serial-scanning'- evidence variable when the eyes move to the right or to the left, evidence of serial scanning can be accumulated until a certain threshold value is obtained. Once the threshold is reached 'serial-scanning' is detected and the system switches from 'chaotic-search' into 'serial-scanning' mode. In 'serial-scanning' mode the system records every tag until a "scan jump" (long distance move above threshold value) is detected which sends the system back into 'chaotic-search' mode.

In detail we used the following threshold values (difference in pixel): ShortHorizontal: < 180, MediumHorizontal: 180-270, LongHorizontal: > 270, ShortVertical: < 50, MediumVertical: 50-120, LongVertical: > 120.

The algorithm identifies 'serial-scanning' when a short or medium horizontal change occurs three times successively without more than one short horizontal 
change. The algorithm was tested semi-formally with a small randomly selected subset of data from different test participants. It provided the same estimation regarding 'serial-scanning' or 'chaotic-search' as a human observer of the data identified in about 70 per cent of cases.

\section{Results}

In the following sections we analyze the observed and recorded gaze behavior of the users with regard to the perception of the tag clouds. We do not focus on the differences in overall search times and user satisfaction, readers interested in these topics are referred to Schrammel et al. [5], where these aspects are reported and discussed in detail.

\subsection{Visual Search Patterns}

\section{Recognizing Alphabetic Tag Organization}

A first relevant question when observing and analyzing the users visual search pattern during interacting with tag clouds is how long it takes the different users to recognize and understand the organization principle of a tag cloud. It is easily possible to identify this point in the eye tracking data for an alphabetic layout and a specific search. Once the user recognizes this organization principle the gaze behavior is much more targeted and heads straightforward to the estimated target position. Unfortunately there is no similar possibility to identify semantic gaze patterns; therefore this section focuses on the alphabetic layout.

We manually analyzed the data provided by the eye tracking system and identified the point at which the users did noticeably change their search behavior. Additionally after the test session we asked the participants if they recognized a layout principle and approximately when. Data from the eye tracking analysis and the self-reported behavior were very stringent, but the data analysis naturally allowed a more finetuned identification of the point in time.

Overall we observed that all participants except one realized the alphabetic order of tags at some time during the experiments. 8 of 15 users recognized the order and adopted the pattern within the first six tags of the target tag search task, 5 of them even within the first three search tasks.

\section{Chaotic and Serial Search Strategies}

Another aim was to identify reading patterns or other search strategies during search task. We could identify two typical search patterns that were used by the participants: chaotic search, which showed no traceable strategy within the search process, and serial scanning, which was typically performed in a characteristic zigzag pattern. These strategies were applied independently from the layout condition i.e. they were equally used for random, semantic and alphabetic layouts. Figure 2 below shows prototypical examples of these two search patterns. 


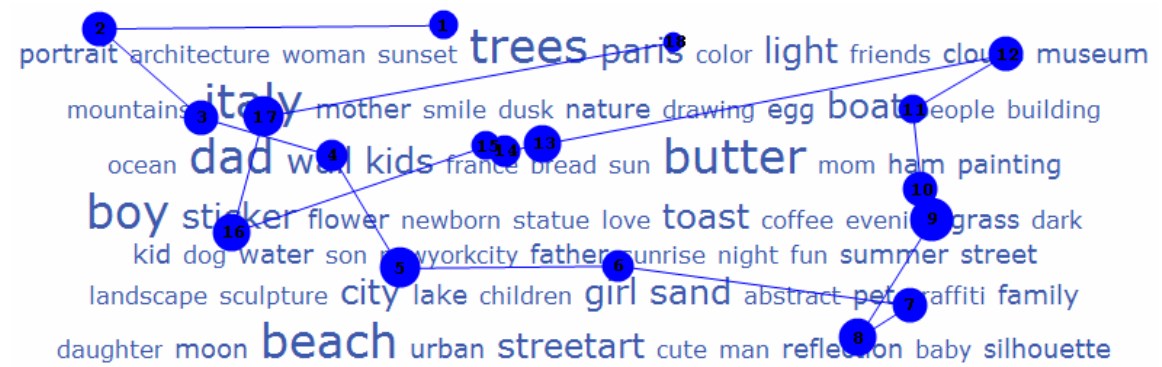

a) Chaotic Search Pattern

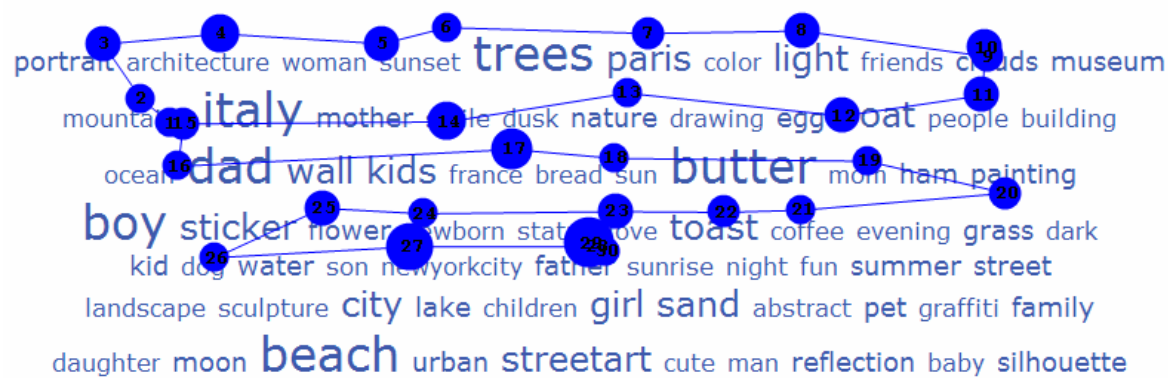

b) Serial Search Pattern (Zigzag)

Fig. 2. Typical Search Patterns

A typical behavior of users is to switch from chaotic to systematic search patterns when the chaotic approach does not turn out to provide any results. We could not identify a clear trend after how much time users change their strategies. There seem to be big differences between users, and also individuals seem to not always apply the same strategy. Please also note that users switched back and forth between the two strategies, and did not always consequently apply serial search until the target was found.

With regard to the scanning tag clouds are almost always scanned in a zigzagpattern, i.e. one line is scanned from left to right, the next from right to left and then starting over from left to right. As described in the methods section we modified a reading detection algorithm that allowed us to automatically detect scanning patterns. According to these algorithm users spend about 70 per cent of the time using chaotic search strategies and 30 percent applying scanning strategies. As mentioned above 10 users scan in both directions, but scanning in reading direction not surprisingly is applied slightly more frequently (57 versus 43 percent of scanning time). Statistical analysis with regard to differences caused by stimulus or task on the ratio of chaotic search versus serial scanning does not show any significant differences.

\subsection{Tag Size and Attention}

Another aspect we were interested in is how the font size of a tag within a tag cloud effects it's perception in the different layout conditions. Common sense and past research [1, 4] suggest that tags in bigger fonts are fixated both more often and for a 
Table 1. Average time a single tag of the regarding size group is looked at

\begin{tabular}{|l|c|c|c|c|c|c|c|c|}
\hline Size & \multicolumn{2}{|c|}{ 3 - very small } & \multicolumn{2}{c|}{ 4 - small } & \multicolumn{2}{c|}{ 5 - big } & \multicolumn{2}{c|}{ 6 - very big } \\
\hline Task & Specific & General & Specific & General & Specific & General & Specific & General \\
\hline Mean & 48,0 & 35,0 & 60,2 & 42,4 & 85,4 & 64,5 & 128,1 & 113,1 \\
\hline
\end{tabular}

longer time than smaller tags. However, it is not clear whether there is a difference of the relative importance of the tag size in different layout conditions as suspected by [5].

Analysis of Variance (ANOVA) with tag size, task type and layout condition as independent variables shows a significant influence of task $\left(\mathrm{F}_{1,19}=5,62, \mathrm{p}=0,028\right)$ and size $\left(\mathrm{F}_{3,61}=69,06 ; \mathrm{p}<0.000\right)$ on the cumulated fixation time per task. The chance for a tag in the biggest font to be fixated by the users gaze is about 2.5 times higher than for a tag in the smallest font. Table 1 below provides an overview on the average time a single tag of the regarding size group was looked at per search task in milliseconds. This trend is very consistent for both types of tasks. Times for the general search tasks are shorter than in specific searches because it is easier to find a tag that qualifies as target.

Schrammel et al. [5] hypothesized that semantic layouts would increase attention towards tags in smaller fonts as they provide valuable information about the semantics of an area of the tag cloud. However, the ANOVA does not show any significant interaction effects $\left(\mathrm{F}_{6,186}=0,32 ; \mathrm{p}=0,92\right)$ for fixation time between tag size and layout condition.

\subsection{Influence of Location and Time on Attention}

In this section we analyze the importance of the tags location in the tag cloud and the time in the search process on the attention of the user. To do so tag clouds were divided into four quadrants for analyzing the data. Time in the search process was dichotomized into initial (first three seconds) and remaining search time. Three seconds for splitting search time was chosen, as an informal interview with 8 experts in human computer interaction showed that this was their average estimation of the typical duration of initial attention of a user in the context of using tag clouds.

Our data show a clear influence of a tags location on the amount of attention it receives from the user. The attention is strongest in the upper-left quadrant and decreases via upper-right and lower-left towards the lower right quadrant. Figure 3 below provides a graphical representation of this data, both for the initial phase of attention and for the whole search task.

ANOVA shows a highly significant effect of quadrant on the amount of visual attention it receives $\left(\mathrm{F}_{3,58}=7,64 ; \mathrm{p}<0.000\right)$. There is no interaction between quadrant and time in the search process $\left(\mathrm{F}_{3,70}=0,32 ; \mathrm{p}=0,811\right)$. This means the attentional preference towards the top and left is not related to effects of initial attention (e.g. users do start at the top left), but that this is a general phenomenon that occurs at all times during the search. 


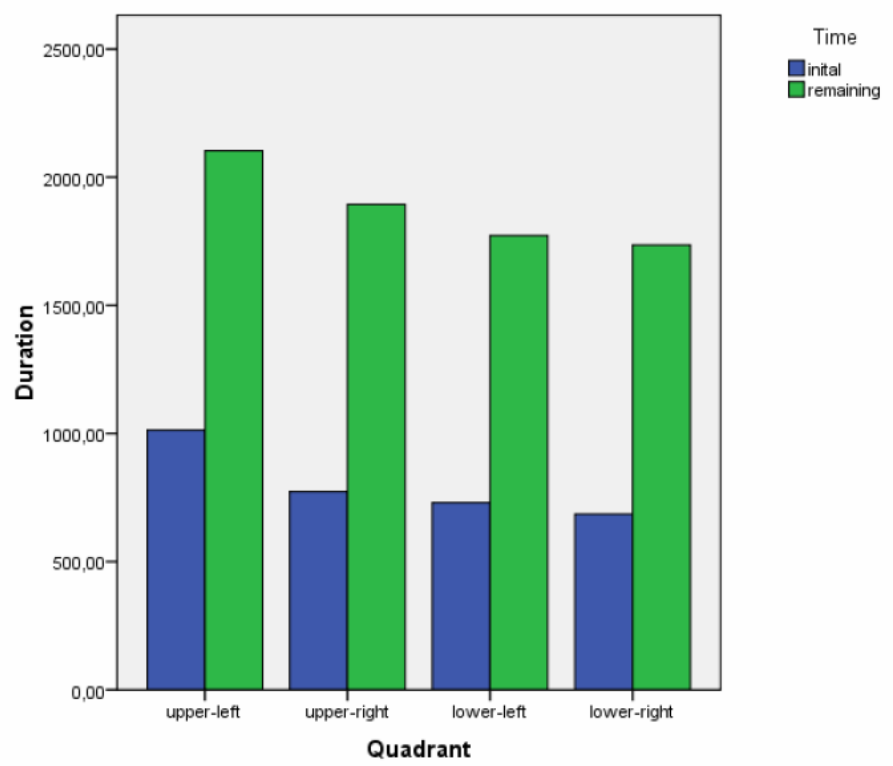

Fig. 3. Attention towards quadrant in initial and remaining time

\subsection{Duration of Fixations}

The duration of a fixation is an indicator for the complexity of a visual task [11]. Complex tasks typically go hand in hand with longer fixations. ANOVA on fixation times shows a significant influence of the task on average duration of fixations $\left(\mathrm{F}_{1,14}=5,08 ; \mathrm{p}=0.041\right)$ : Fixations typically were longer for general search tasks than for specific searches. This is easy to understand, because in the general search the user not only has to identify but also classify the focused tag to decide whether it is a correct choice or not. With regard to the layout conditions we hypothesized the fixation duration to be longest in the semantic condition as the understanding and processing of the semantic layout probably would add additional cognitive load. As expected this is the case for both tasks. However, this difference is not statistically significant $\left(\mathrm{F}_{2,28}=1,82 ; \mathrm{p}=0,180\right)$.

\section{Discussion}

In this paper we described the results of our analysis of the visual perception of tag clouds. In accordance with prior research tag size was identified as the major influence on the perception. Through our research we were able to clarify conflicting issues with regard to the influence of a tags position on its perception: There is a clear and consistent influence of the quadrant on the amount of attention a tag can expect. The eye tracking analysis showed clearly that the attention decreases from top to bottom as well as left to right. 
We did not find any relevant differences in attention between different tag layouts. This was very surprising to us, as for the case of semantic layouts we expected an increased focus on tags in smaller size, as they convey important information about the thematic area of a tag cloud. Also we expect to be able to identify differences in the search patterns related to the organization principle. From the qualitative interviews we know that not recognizing the thematic layout cannot be the sole source of this indifference. Also the reported significant differences in search times between semantic and random layouts suggest that there are different perceptual processes at work. However, these differences do not seem to be related to the overall amount of attention tags in different sizes receive or the application of recognizably different search strategies. We think the explanation might be that users learn the topical organization of a semantic tag cloud implicitly, and therefore no explicit change in the behavior can be observed. Another possible explanation is that users are able to identify the topic of an area without the need to focus on all the different words but by a blurry perception. However, provided that the visual capabilities of humans is only $2^{\circ}$ or 35 pixel as described above we do not champion this explanation.

\section{Conclusions}

In this paper we could identify (and clarify) several important issues with regard to the visual perception of tag clouds. In future work we plan to further explore influences of different aspects such as number of tags in the tag cloud, familiarity with topic area and visual designation of semantic areas on the perception.

Aknowledgements. This work was supported by the Softnet Austria Competence Network. We also would like to thank Rainer Pfister for implementing the scanning detection algorithm.

\section{References}

1. Rivadeneira, A.W., Gruen, D.M., Muller, M.J., Millen, D.R.: Getting our head in the clouds: toward evaluation studies of tagclouds. In: Proc. CHI 2007, pp. 995-998. ACM Press, New York (2007)

2. Maughan, L., Gutnikov, S., Stevens, R.: Like more, look more. Look more, like more: The evidence from eye-tracking. Journal of Brand Management 14 (2007)

3. Halvey, M.J., Keane, M.T.: An assessment of tag presentation techniques. In: Proc. WWW 2007, pp. 1313-1314. ACM Press, New York (2007)

4. Bateman, S., Gutwin, C., Nacenta, M.: Seeing things in the clouds: the effect of visual features on tag cloud selections. In: Proc. Hypertext and Hypermedia 2008, pp. 193-202. ACM Press, New York (2008)

5. Schrammel, J., Leitner, M., Tscheligi, M.: Semantically Structured Tag Clouds: An Empirical Evaluation of Clustered Presentation Approaches. In: CHI 2009, Boston, MA, USA, April 3-9 (2009)

6. Hassan-Montero, Y., Herrero-Solana, V.: Improving tagclouds as visual information retrieval interfaces. In: Proc. InfoSciT 2006 (2006) 
7. Fujimura, K., Fujimura, S., Matsubayashi, T., Yamada, T., Okuda, H.: Topigraphy: visualization for largescale tag clouds. In: Proc. WWW 2008, pp. 1087-1088. ACM Press, New York (2008)

8. Berlocher, I., Lee, K., Kim, K.: TopicRank: bringing insight to users. In: Proc. SIGIR 2008, pp. 703-704. ACM Press, New York (2008)

9. Sinclair, J., Cardew-Hall, M.: The Folksonomy Tag Cloud: When is it Useful? Journal of Information Sience 34(1), 15-29 (2008)

10. Kuo, B.Y., Hentrich, T., Good, B.M., Wilkinson, M.D.: Tag clouds for summarizing web search results. In: Proc. World Wide Web 2007, pp. 1203-1204. ACM Press, New York (2007)

11. Rayner, K.: Eye movements in reading and information processing: 20 years of research. Psychological Bulletin 124, 372-422 (1998)

12. Goldberg, H.J., Kotval, X.P.: Computer interface evaluation using eye movements: Methods and constructs. International Journal of Industrial Ergonomics 24, 631-645 (1999)

13. Posner, M.I.: Orienting of attention. Quarterly Journal of Experimental Psychology 32, 3 $25(1980)$

14. Duchowski, A.T.: A Breadth-First Survey of Eye Tracking Applications. Behavior Research Methods, Instruments, and Computers 34(4), 455-470 (2002)

15. Carpenter, P.A., Shah, P.: A model of perceptual and conceptual processes in graph comprehension. Journal of Experimental Psychology: Applied 4, 75-100 (1998)

16. Klöckner, K., Wirschum, N., Jameson, A.: Depth and breadth-first processing of search result lists. In: Ext. Abstracts CHI 2004, pp. 1539-1539. ACM Press, New York (2004)

17. Cutrell, E., Guan, Z.: What are you looking for? An eye-tracking study of information usage in Web search. In: CHI 2007- Proceedings - Gaze \& Eye Tracking, San Jose, California, USA, April 28 - May 3 (2007)

18. Pan, B., Helmbrooke, H.A., Gay, G.K., Granka, L.A., Feusner, M.K., Newman, J.K.: The Determinants of Web Page Viewing Behavior: An Eye Tracking Study. In: Proceedings of Eye Tracking Research \& Applications: Symposium 2004, pp. 147-154 (2004)

19. Lohse, G.L.: Consumer eye movement patterns on Yellow Pages advertising. Journal of Advertising 26, 61-73 (1997)

20. Rayner, K., Rottello, C.M., Stewart, A.J., Keir, J., Duffy, S.A.: Integrating Text and Pictorial Information: Eye Movements when Looking at Print Advertisements. Journal of Experimental Psychology: Applied 7, 219-226 (2001)

21. Flickr API, http://www.flickr.com/services/api

22. http://forrest.psych.unc.edu/research/alscal.html

23. Campbell, C.S., Maglio, P.P.: A robust algorithm for reading detection. In: Proceedings of the ACM Workshop on Perceptual User Interfaces (2001) 